\title{
INCIDENCE STRATIFICATIONS ON HILBERT SCHEMES OF SMOOTH SURFACES, AND AN APPLICATION TO POISSON STRUCTURES
}

\begin{abstract}
ZIV RAN
ABSTRACT. Given a smooth curve on a smooth surface, the Hilbert scheme of points on the surface is stratified according to the length of the intersection with the curve. The strata are highly singular. We show that this stratification admits a natural log-resolution, namely the stratified blowup. As a consequence, the induced Poisson structure on the Hilbert scheme of a Poisson surface has unobstructed deformations.
\end{abstract}

One of the important and well-studied geometric objects associated to a smooth surface $X$ is the Hilbert scheme $X^{[\ell]}$, parametrizing 0-dimensional subschemes of length $\ell$ on $X$. This is a smooth $2 \ell$-dimensional variety, which inherits various aspects of the geometry of $X$, e.g. a symplectic structure [1]. See [3], [7] for information and references on Hilbert schemes.

Now suppose one is interested not in the 'plain' surface $X$ but rather in a pair $(X, Y)$, where $Y$ is a smooth curve on $X$. To this one can quite analogously associate a stratification, called an incidence stratification

$$
Y^{(\ell)}=I_{Y}^{\ell} \subset I_{Y}^{\ell-1} \subset \ldots \subset I_{Y}^{1} \subset X^{[\ell]}
$$

where the closed stratum $I_{Y}^{j}$ denotes the locus of schemes intersecting $Y$ in length at least $j$. Though natural enough, this stratification unfortunately seems to have somewhat complicated singularities except for the bottom stratum $I_{Y}^{\ell}$ : e.g. for $\ell=2, I_{Y}^{1}$ has Whitney-umbrella type singularities along $I_{Y}^{2}$. Things get still more complicated in a neighborhood of worse-behaved, e.g. non-curvilinear schemes. Thus, one is led to try to resolve the singularities of this stratification in a simple and natural way.

Given that $I^{\ell}=Y^{(\ell)}$ is smooth, the simplest potential way to resolve the singularities of the incidence stratification is by 'stratified blowup': i.e. blow up $I_{Y}^{\ell}$, then blow up the proper transform of $I_{Y}^{\ell-1}$, etc. The purpose of this paper is to show that the stratified blowup indeed resolves the singularities of the incidence stratification.

Date: September 10, 2018.

2010 Mathematics Subject Classification. 14C05, 14J99, 32J15, $53 \mathrm{D} 17$.

Key words and phrases. Complex surface, Hilbert scheme, stratification, normal crossings, Poisson structure,

arxiv.org/1502.00553. 
This question, of independent interest, first arose in connection with Poisson structures on Hilbert schemes of projective Poisson (i.e. anticanonical) surfaces (see [4], [6]), and indeed our result has some applications to such structures and their deformations, see Corollary 5 ,

Here we begin in $\S 1$ by proving an analogous stratification result for loci of collections of univariate polynomials stratified by the number of their common zeros. Then in $\$ 2$ we will prove the main result in a neighborhood of a monomial ideal. Finally in $\$ 3$ we will prove the general case by specializing a general ideal to a monomial one.

In this paper we work over an algebraically closed field $\underline{k}$ of arbitrary characteristic.

We heartily thank the referee for his careful reading of the paper and for many corrections and helpful comments.

\section{UNIVARIATE POLYNOMIALS WITH MANY COMMON ZEROS}

Our main theorem depends on a completely elementary result about a stratification in certain spaces of polynomials in 1 variable, which corresponds to the number of common zeros of polynomials.

Fix natural numbers $m_{1}, \ldots, m_{n}$ and set $m=m_{1}+\ldots+m_{n}$. Consider the space $\mathbb{A}=$ $\mathbb{A}\left(m_{1}, \ldots, m_{n}\right)$ of $2 n$ - tuples $\left(h_{1}, \ldots, h_{n}, f_{1}, \ldots, f_{n}\right)$ of polynomials (coefficients in $\underline{k}$ ) of the form

$$
\begin{aligned}
& h_{i}=x^{m_{i}}+\sum_{j=0}^{m_{i}-1} b_{i, j} x^{j}, \\
& f_{i}=\sum_{j=0}^{m_{i}-1} a_{i, j} x^{j}, \quad i=1, \ldots, n .
\end{aligned}
$$

This is clearly an affine space of dimension $2 m$; in fact, it can be identified with a linear space with origin $0=\left(x^{m_{1}}, \ldots, x^{m_{n}}, 0, \ldots, 0\right)$. Set

$$
h=\prod_{i=1}^{n} h_{i}, p_{i}=f_{i} \prod_{j \neq i} h_{j} .
$$

Thus $h$ is monic of degree $m$ exactly and each $p_{i}$ is of degree at most $m-1$. For each $k=m, m-1, \ldots, 1$, consider the locus $I^{k} \subset \mathbb{A}$ consisting of all $(h ., f$.) such that the ideal generated by $h, p_{1}, \ldots, p_{n}$ has colength at least $k$. Thus we have a chain of closed subschemes, i.e. a stratification

$$
I^{m} \subset I^{m-1} \subset \ldots \subset I^{1} \subset \mathbb{A} .
$$

Consider the associated stratified blowup, i.e. the blowup $\hat{\mathbb{A}}$ of $\mathbb{A}$ obtained by first blowing up $I^{m}$, then the proper transform of $I^{m-1}$, etc. 
Proposition 1. Near the origin, for each $j=1, \ldots, m$, the proper transform $\hat{I}^{j}$ of $I^{j}$ in $\hat{A}$ is smooth and the total transform equals $\hat{I}^{m}+\ldots+\hat{I}^{j}$ and is a divisor with normal crossings.

Proof. To begin with, $I^{m}$ is defined by the vanishing of the $a_{i, j}, j=0, \ldots, m_{i}-1, i=1, \ldots, n$, hence is smooth of codimension $m$. Hence the blowup $\mathbb{A}_{1}$ of $\mathbb{A}$ in $I^{m}$ is smooth, and is covered by open affines where some $a_{i, j} \neq 0$. Now on $\mathbb{A}_{1}$, the intersection of the exceptional divisor with the proper transform of $I^{m-1}$ is covered by open affines $U_{i}^{1}$ where $a_{i, m_{i}-1} \neq 0$ for some $i$, and on this open set, the proper transform of $I^{m-1}$ is defined by the equations

$$
a_{k, j}=0, \forall k \neq i, j=0, \ldots, m_{k}-1,
$$

plus the equation

$$
f_{i}^{1}:=\operatorname{Rem}\left(h_{i}, f_{i}\right)=0
$$

where $\operatorname{Rem}(u, v)$ denotes remainder dividing $u$ by $v$; thus in this case,

$$
f_{i}^{1}=h_{i}-q_{i} f_{i}, q_{i}:=\frac{1}{a_{i, m_{i}-1}}\left(x+b_{i, m_{i}-1}-a_{i, m_{i}-2} / a_{i, m_{i}-1}\right) \text {. }
$$

Note that the above formulas establish an isomorphism between the set of pairs $\left(f_{i}, h_{i}\right)$ as above (an open set in an affine space) and the space of triples $\left(f_{i}^{1}, q_{i}, f_{i}\right)$ where

$$
\operatorname{deg}\left(f_{i}^{1}\right) \leq m_{i}-2, f_{i}=a_{i, m_{i}-1} x^{m_{i}-1}+\text { (lower), } q_{i}=\frac{1}{a_{i, m_{i}-1}} x+c ; a_{i, m_{i}-1} \neq 0, c \in \underline{k}, .
$$

It follows in particular that the locus defined by $f_{i}^{1}=0$ is nonsingular of codimension $m_{i}-2$. Write

$$
f_{i}^{1}=\sum_{j=0}^{m_{i}^{1}-1} a_{i, j}^{1} x^{j}, m_{i}^{1}=m_{i}-1,
$$

Also set

$$
f_{k}^{1}:=h_{k}, a_{k, j}^{1}=a_{k, j}, m_{k}^{1}=m_{k}, k \neq i .
$$

Thus with (1) and (2) we get in all a total of $m-1=\sum_{k=1}^{n} m_{k}^{1}$ equations with independent differentials, namely

$$
a_{k, j}^{1}=0, j=0, \ldots, m_{k}^{1}-1, k=1, \ldots, n,
$$

defining the proper transform of $I^{m-1}$, so this proper transform is smooth here and transverse to the exceptional divisor, which has equation $a_{i, m_{i}-1}=0$.

Next we blow up the proper transform of $I^{m-1}$, thus obtaining $\mathbb{A}_{2}$ which is smooth, and has smooth exceptional divisor over $\mathbb{A}_{1}$. Notice that the intersection of the exceptional divisor of $\mathbb{A}_{2}$ over $\mathbb{A}_{1}$ with the proper transform of $I^{m-2}$ is covered by open sets $U_{i}^{1} U_{k}^{2}$ 
where the leading ( $m_{i}$-th) coefficient of some $f_{i}$ and the leading ( $m_{k}^{1}$-th) coefficient of some $f_{k}^{1}$ are both nonzero, $i, k=1, \ldots, n$ distinct or not, and on $U_{i}^{1} U_{k}^{2}$ the proper transform of $I^{m-2}$ is defined by the vanishing of (the coefficients of) $f_{k}^{2}:=\operatorname{Rem}\left(h_{k}, f_{k}^{1}\right.$ ) (which is $m_{k}^{2}:=m_{k}^{1}-1$ many equations with independent differentials), plus the vanishing of $f_{d}^{2}:=f_{d}^{1}, \forall d \neq k$. Then we can continue in the same way.

\section{NeAR A MONOMial IDEAL}

In this section we will prove a special case of our main theorem in a neighborhood of a monomial ideal cosupported at a point.

Let $(s)=.\left(s_{1}, \ldots, s_{n}\right),(t)=.\left(t_{1}, \ldots, t_{n}\right)$ be sequences of nonnegative integers. To these we associate the planar ideal

$$
\mathfrak{a}:=\mathfrak{a}(s ., t .)=A G_{0}+\ldots+A G_{n}, A:=\underline{k}[x, y], G_{i}:=x^{t_{1}+\ldots+t_{n-i}} y^{s_{1}+\ldots+s_{i}}, i=0, \ldots, n .
$$

We will assume this is cosupported at the origin, i.e. that $\left(\sum t_{i}\right)\left(\sum s_{i}\right)>0$. In this case the ideal is of finite colength equal to

$$
L=\sum_{i+j \leq n+1} s_{i} t_{j}
$$

As is often the case, $\mathfrak{a}$ can be usefully represented as a determinantal ideal, namely as the ideal of $n \times n$ minors of the $n \times(n+1)$ matrix $M=\left(m_{i, j}\right)$ where

$$
m_{i, j}=\left\{\begin{array}{l}
x^{t_{i}}, j=i \\
y^{s_{n+1-i}}, j=i+1 ; \\
0, \text { otherwise. }
\end{array}\right.
$$

Thus

$$
M=\left[\begin{array}{cccccc}
x^{t_{1}} & y^{s_{n}} & 0 & 0 & \ldots & 0 \\
0 & x^{t_{2}} & y^{s_{n-1}} & 0 & \ldots & 0 \\
& & \vdots & & & \\
0 & \ldots & 0 & 0 & x^{t_{n}} & y^{s_{1}}
\end{array}\right] .
$$

Thus $G_{i}=\operatorname{det}\left(M_{i}\right)$ where $M_{i}$ is the submatrix of $M$ obtained by deleting the $(n+1-i)$-th column. Accordingly, a admits the short resolution

$$
0 \rightarrow A^{n} \stackrel{t^{M}}{\rightarrow} A^{n+1} \stackrel{(G .)}{\rightarrow} \mathfrak{a} \rightarrow 0
$$

where $(G)=.\left(G_{n},-G_{n-1}, \ldots,(-1)^{n} G_{0}\right)$.

Let $\mathcal{H}$ denote the Hilbert scheme of colength - $L$ ideals in $\underline{k}[x, y]$, an open subset of the Hilbert scheme of $\mathbb{P}^{2}$. By Fogarty's theorem (see [2] or [3] or [7], Theorem 4.6.9, p.248), 
$\mathcal{H}$ is smooth at the point corresponding to $\mathfrak{a}$, and has tangent space $\operatorname{Hom}(\mathfrak{a}, \underline{k}[x, y] / \mathfrak{a})$ so the latter vector space has dimension $2 L$. For a quick proof of Fogarty's theorem, use the exact sequence

$$
0 \rightarrow \mathfrak{a} \rightarrow \underline{k}[x, y] \rightarrow \underline{k}[x, y] / \mathfrak{a} \rightarrow 0
$$

to see that $\operatorname{Ext}^{1}(\mathfrak{a}, \underline{k}[x, y] / \mathfrak{a}) \simeq \operatorname{Ext}^{2}(\underline{k}[x, y] / \mathfrak{a}, \underline{k}[x, y] / \mathfrak{a})$, which is Serre dual to $\operatorname{Hom}(\underline{k}[x, y] / \mathfrak{a}, \underline{k}[x, y] / \mathfrak{a})$, hence is $L$-dimensional, and morevoer that

$$
\chi(\mathfrak{a}, \underline{k}[x, y] / \mathfrak{a})=\operatorname{dim}(\operatorname{Hom}(\mathfrak{a}, \underline{k}[x, y] / \mathfrak{a}))-\operatorname{dim}\left(\operatorname{Ext}^{1}(\mathfrak{a}, \underline{k}[x, y] / \mathfrak{a})\right)
$$

is locally constant on $\mathcal{H}$, being an Euler characteristic.

Now as is well known, local deformations of $\mathfrak{a}$ are obtained by deforming the matrix $M$, i.e. replacing $M$ by $\tilde{M}=M+N$, where $N$ can be taken with coefficients in $(A / \mathfrak{a}) \otimes \mathfrak{n}$, where $S=\underline{k} \oplus \mathfrak{n}$ is a local $\underline{k}$-algebra. In the case of infinitesimal deformations, $S$ is artinian, i.e. finite-dimensional. This well-known fact can be proved as follows. Given an $S$-flat ideal $\tilde{\mathfrak{a}}<A \otimes S$, lifting the $G$ generators yields a map $\tilde{G}: A^{n+1} \otimes S \rightarrow \tilde{\mathfrak{a}}$. The kernel $\tilde{K}=\operatorname{ker}(\tilde{G})$ is also $S$-flat and has $\tilde{K} \otimes(S / \mathfrak{n})=\operatorname{ker}(G$. $) \simeq A^{n}$. Therefore $\tilde{K}$ itself is free so $\tilde{K} \simeq A^{n} \otimes S$.

Let $t=t_{1}+\ldots+t_{n}$ and note that $\mathfrak{a} . \underline{k}[x]=\mathfrak{a} / \mathfrak{a} \cap(y)$ is an ideal of colength $t$. For $k=1, \ldots, t$ let $I_{\mathfrak{a}}^{k}$ denote the subscheme of $\mathcal{H}_{\mathfrak{a}}$, the germ of $\mathcal{H}$ at $\mathfrak{a}$, consisting of deformations of $\mathfrak{a}$ whose image in $k[x]$ is of colength at least $k$; the scheme structure on $I_{\mathfrak{a}}^{k}$ can be defined via a suitable Fitting ideal associated to the restriction of (4) on the $x$-axis. Thus, we have a stratification by closed subschemes

$$
I_{\mathfrak{a}}^{t} \subset I_{\mathfrak{a}}^{t-1} \subset \ldots \subset I_{\mathfrak{a}}^{1} \subset \mathcal{H}_{\mathfrak{a}} .
$$

Let $\hat{\mathcal{H}}_{\mathfrak{a}}$ denote the corresponding stratified blowup.

Proposition 2. For each $k=t, \ldots, 1$, the proper transform $\hat{I}_{\mathfrak{a}}^{k}$ of $I_{\mathfrak{a}}^{k}$ in $\hat{\mathcal{H}}_{\mathfrak{a}}$ is smooth and the total transform equals $\hat{I}_{\mathfrak{a}}^{t}+\ldots+\hat{I}_{\mathfrak{a}}^{k}$ and is a divisor with normal crossings.

Proof. Write the main diagonal and last column elements of $\tilde{M}$ as

$$
\begin{aligned}
(\tilde{M})_{i, i}=h_{i}=x^{t_{i}}+\sum b_{i, j} x^{j},\left(\tilde{M}_{i, n+1)}\right. & =f_{i}=\sum a_{i, j} x^{j}, i<n, \\
\left(\tilde{M}_{n, n+1)}\right. & =y^{s_{1}}+f_{n}=y^{s_{1}}+\sum a_{n, j} x^{j} .
\end{aligned}
$$

Note that the deformations corresponding to $a_{i, j} x^{j}, b_{i, j} x^{j}$ are linearly independent and together these yield a $2 t$-dimensional subvariety of $\hat{\mathcal{H}}_{a}$, which maps isomorphically to the space $\mathbb{A}$ considered in the previous sections. Moreover, given a deformation of $\mathfrak{a}$ corresponding to $\tilde{M}$, its restriction on the $x$-axis is determined by

$$
h=\prod_{5} h_{i}
$$


which is the deformation of $G_{0}$, and by

$$
p_{i}=f_{i} \prod_{j \neq i} h_{j},
$$

which is the deformation of $G_{n+1-i}, i=1, \ldots, n$. Consequently, our result follows from Proposition 1 .

\section{INCIDENCE STRATIFICATIONS: GENERAL CASE}

We are now ready to state and prove the main result. Thus, let $Y$ be a smooth closed curve on the smooth algebraic surface $X$ over an algebraically closed field $\underline{k}$. For simplicity, we (needlessly) assume $X$ quasi-projective, but see the remarks following the proof. Let $I_{Y}^{k} \subset X^{[\ell]}$ denote the subscheme of the length- $\ell$ Hilbert scheme of $X$ consisting of schemes whose intersection with $Y$ is of length $k$ or more. $I_{Y}^{k}$ may be endowed with a scheme structure as the image of a natural closed subscheme of the flag Hilbert scheme $X^{[k, \ell]}$ (see [7]) that is the pullback of the closed subscheme $Y^{[k]} \subset X^{[k]}$ under the natural map $X^{[k, \ell]} \rightarrow X^{[k]}$. We thus have a closed stratification, called the incidence stratification associated to $Y$ :

$$
Y^{(\ell)}=I_{Y}^{\ell} \subset I_{Y}^{\ell-1} \subset \ldots \subset I_{Y}^{1} \subset X^{[\ell]} .
$$

It is easy to see that each closed stratum $I_{Y}^{k}$ has codimension $k$ in $X^{[\ell]}$.

Theorem 3. In the stratified blowup of the incidence stratification, the proper transform $\hat{I}_{Y}^{k}$ of each closed stratum $I_{Y}^{k}$ is smooth and the total transform equals $\hat{I}_{Y}^{k}+\hat{I}_{Y}^{k+1}+\ldots+\hat{I}_{Y}^{t}$ and has normal crossings.

Proof. The statement is local near a given point $z \in X^{[\ell]}$. Further, because of the usual étale, or analytic product decomposition of the Hilbert scheme corresponding to the support of $z$, we may assume $z$ is supported in a single point. Thus, we may work locally and assume $X$ is the plane $\mathbb{A}^{2}$ and $Y$ is the $x$-axis with ideal $(y)$ and $z$ is supported at the origin. Note that the 'punctual' Hilbert scheme $X_{0}^{[\ell]}$ consisting of subschemes supported at the origin is projective. Consider the action of the multiplicative group $\mathbb{G}_{\mathrm{m}}$ on $X^{[\ell]}$ and $X_{0}^{[\ell]}$ induced by the action on $X$ given by $y \mapsto \lambda$.y (fixing $x$ ). Viewing $\mathbb{G}_{\mathrm{m}}$ as subset of $\mathbb{A}^{1}$, let

In other words, the map

$$
z_{0}=\lim _{\substack{\lambda \in \mathrm{m}_{\mathrm{m}} \\ \lambda \rightarrow 0}} \lambda^{*} z \in X_{0}^{[\ell]} .
$$

$$
f_{0}: \mathbb{G}_{\mathrm{m}} \rightarrow X_{0}^{[\ell]}, f_{0}(\lambda)=\lambda^{*} z,
$$

extends by projectivity to a map $f: \mathbb{A}^{1} \rightarrow X_{0}^{[\ell]}$ and $z_{0}=f(0)$. Then $z_{0}$ is $\mathbb{G}_{\mathrm{m}}$-invariant, i.e. a homogeneous ideal with respect to $y$. Let $R=O_{Y, 0}$, which is DVR with parameter $x$. Then 
the ideal $z_{0} R[y]$ in $R[y]$ is homogeneous as well with respect to $y$, hence is generated by finitely many homogeneous elements of the form $a y^{s}, s \geq 0, a \in R$. Adjusting by a unit, we may assume $a=x^{r}, r \geq 0$. Because $z_{0}=\left(z_{0} R[y]\right) \cap \underline{k}[x, y]$, it too is generated by such elements, i.e. $z_{0}$ is in fact a monomial ideal. By Proposition 2, the Theorem holds in a neighborhood of $z_{0}$. Moreover, every neighborhood of $z_{0}$ in $X^{[\ell]}$ contains schemes equivalent to $z$. Therefore the theorem holds locally near these as well, hence locally near $z$.

Remarks 4. (i) Because Theorem 3 is local in nature, it actually holds without any quasiprojectivity hypotheses on $X$. First, the Hilbert scheme $X^{[\ell]}$ exists as a scheme (for any algebraic scheme $X$ ), and can be constructed as a projective morphism (cycle map) over the symmetric product $X^{(\ell)}$, which itself can be covered by patches which are cartesian products of symmetric products of quasi-projective schemes. See for instance [5], §1. Second, the scheme structure on $I_{Y}^{k}$ can be constructed patch-wise from the quasi-projective case. Lastly, the main argument of the proof is local around a scheme supported on a single point, so certainly carries over.

(ii) The obvious analogue of Theorem 3 in the complex-analytic category holds with the same proof, mutatis mutandis.

As explained in [4] and in [6], Example 4.3, Theorem 3 has an application to the deformation theory of induced Poisson structures on Hilbert schemes of Poisson surfaces (which in turn is an analogue of a result of Voisin [8] in the case of symplectic structures):

Corollary 5. Let $\Pi$ be a Poisson structure on a smooth complex projective surface $S$, corresponding to a smooth anticanonical curve, and let $\Pi^{[r]}$ be the associated Poisson structure on the Hilbert scheme $S^{[r]}$. Then the pair $\left(S^{[r]}, \Pi^{[r]}\right)$ has unobstructed deformations.

Proof. In light of the argument in loc. cit. this almost follows from normal crossings of $\hat{I}^{r}+\ldots+\hat{I}^{1}$, which coincides with the inverse image $\tilde{D}$ of the Pfaffian divisor of $\Pi$ on the stratified blowup $\hat{S}^{[r]}$. The only missing point is that $\Pi$ lifts holomorphically to $\hat{S}^{[r]}$. This can be checked locally at a generic point of each $\hat{I}^{k}$. Now it is clear from our proof above that such a generic point corresponds to a reduced scheme, i.e. $r$ distinct points on $S$, of which exactly $k$ are on $C=[\Pi]$. There, $\Pi^{[r]}$ can be written locally as

$$
y_{1} \partial x_{1} \wedge \partial y_{1}+\ldots+y_{k} \partial x_{k} \wedge \partial y_{k}+\partial x_{k+1} \wedge \partial y_{k+1}+\ldots+\partial x_{r} \wedge \partial y_{r} .
$$

On a suitable (typical) open set in the blowup, we can write locally $y_{i}=u_{i} y_{1}, i=2, \ldots, k$ so clearly $\partial y_{i}$ has a pole of at most $y_{1}$, which is cancelled by $y_{i}$, so $\Pi^{[r]}$ is holomorphic. 


\section{REFERENCES}

1. A. Beauville, Variétés Kählériennes dont la première classe de Chern est nulle, J. Differential Geometry $\mathbf{1 8}$ (1983), 755-782.

2. J. Fogarty, Algebraic families on an algebraic surface, Amer. J. Math. 90 (1968), 511-521.

3. M. Lehn, Lectures on Hilbert schemes, CRM notes, Centre de Recherches Mathématiques, Montreal, 2004.

4. Z. Ran, Deformations of holomorphic pseudo-symplectic Poisson manifolds, arxiv.org/1308.2442.

5. __ Structure of the cycle map for Hilbert schemes of families of nodal curves, Israel J. Math., 1-34, (to appear), http: //arXiv.org/0903.3693.

6. - Deformations of log-Lagrangian submanifolds of Poisson manifolds, Int. Math. Research Notices (2014), arxiv.org/1311.2656.

7. E. Sernesi, Deformations of algebraic schemes, Grundl. d. math. Wiss., vol. 334, Springer International, Berlin, Heidelberg, 2006.

8. C. Voisin, Sur la stabilité des sous-variétés lagrangiennes des variétés symplectiques holomorphes, Complex projective geometry (G. Ellingsrud, C. Peskine, G. Sacchiero, and S.A. Stromme, eds.), Cambridge university press, 1992, pp. 294-303.

UC MATH DEPT.

BIG SPRINGS ROAD SURGE FACILITY

RIVERSIDE CA 92521 US

ZIV.RAN @ UCR.EDU

HTTP : / /MATH.UCR.EDU/ ZIV/ 